\title{
Novel detection of mutation in the TECPR2 gene in a Chinese hereditary spastic paraplegia 49 patient: a case report
}

\author{
Yalin Guan ${ }^{1,2^{*}} \mathbb{D}$, Hui Lu ${ }^{1,2}$, Wenchao Zuo ${ }^{1,2}$, Xiaodan Wang ${ }^{1,2}$, Shimin Wang ${ }^{1,2}$, Xinping Wang ${ }^{1,2}$, Feng Liu ${ }^{1,2}$, \\ Kun Jia ${ }^{1,2}$, Rui Gao ${ }^{1,2}$, Hao Wu ${ }^{1,2}$, Zhihong Shi ${ }^{1,2}$ and Yong Ji, ${ }^{1,2}$
}

\begin{abstract}
Background: Hereditary spastic paraplegia 49 (HSP49) is an autosomal recessive genetic disease first discovered in 2012; and which the mutation primarily affects Bukharian Jewish patients.

Case presentation: The present case reports the first instance of HSP49 detected in China. The patient had normal mental development and good athletic ability before 10 years old and presented with instable temperature, mental retardation, spastic ataxia, and paroxysmal convulsions. Genetic diagnosis was based on detection of whole exons and two heterozygous variants in the exon region of the TECPR2 gene: c.1729C > T and C.4189G > A. Mutations at these two sites have not been previously reported.

Conclusions: This case expands the gene mutation spectrum and clinical phenotypic characteristics of autosomal recessive HSP in China; moreover, it indicates differences in the clinical phenotype of HSP49 in different ethnicities. In addition, this reported provides further evidence regarding the effectiveness of targeted next-generation sequencing technology in improving the efficiency and diagnostic rate of genetic diagnosis of HSP.
\end{abstract}

Keywords: Hereditary spastic paraplegia, Autosomal recessive inherited disease, Spastic ataxia, Heterozygous mutations, Case report

\section{Background}

Hereditary spastic paraplegia (HSP) is a group of familial diseases characterized by progressive degeneration of the corticospinal tract. The emergence of next-generation sequencing has allowed recognition of the extreme variability of genes and mutations in HSP [1, 2]. The inheritance mode of HSP includes autosomal dominant inheritance, autosomal recessive inheritance, and $\mathrm{X}$-linked inheritance [1]. Its gene locus is termed spastic paraplegia [3], and it is numbered sequentially. To date,

\footnotetext{
*Correspondence: guanyalintj@126.com

1 Department of Neurology, Tianjin Huanhu Hospital, Tianjin 300350, China

Full list of author information is available at the end of the article
}

the number of discovered pathogenic gene loci exceeds 83 and continues to increase [4].

Hereditary spastic paraplegia 49 (HSP49) was first reported by Oz-Levi et al. in 2012 [5] and is an autosomal recessive inherited disease caused by mutations in the TECPR2 gene. HSP49 mainly manifests as recessive inherited psychomotor retardation, mental retardation, spastic ataxia, and recurrent apnea. Previous reports indicated that HSP49 was due primarily to homozygous mutations in a Bukharian Jewish population [5] and heterozygous mutations in non-Bukharian Jewish populations [6, 7]. Most HSP49 cases have been reported in Israel, while no cases have been officially reported in China. This article reports a case of a Chinese patient with HSP49 caused by a heterozygous gene mutation. 


\section{Case presentation}

The patient in the present case was a 19-year-old male. His parents are of Chinese descent and are healthy and not consanguineous. They were not reported in his extended family. He was born via cesarean section at 39 weeks of gestation in March 2001 with a birthweight of $3200 \mathrm{~g}$. Before 10 years of age, the patient's intelligence and athletic ability were unaffected. He had excellent performance in an English speech contest and in swimming.

In February 2011, at the age of 10 years old, a high fever persisted for 4 days, after which an epileptic seizure occurred several times in one day; one day later, the patient exhibited a persistent loss of consciousness and dyspnea. Following treatment with antiepileptic drugs, glucocorticoid, plasma exchange, and endotracheal intubation, the patient's symptoms were controlled. However, the patient exhibited the following sequelae: severe cognitive impairment, personality change, and aphasia.

In the following 9 years, the patient experienced frequent recurrent seizures. Moreover, he presented a paroxysmal temperature increase 1-2 times daily during which body temperature suddenly increased from $36^{\circ} \mathrm{C}$ to higher than $38^{\circ} \mathrm{C}$ without infective symptoms and which self-remitted after a few hours. The walking ability of the patient gradually decreased, and in 2018, he needed wheelchair or walking with assistance from his parents. He had normal digestive function without acid reflux heartburn symptoms; he rarely had pulmonary infection. The patient slept normally at night and had no abnormal breathing during sleep.

Three months before admission, on February 2020, his caregiver forced him to take prescribed medicine because he refused to take the medicine orally; this led to a repeated cough, choking, dysphagia, aspiration pneumonia, and an increase in limb seizures, which occurred about 10 times per day, each lasting 1-10 min. At admission, the patient was in a moderate coma. Pupils were round and reactive to light. The neck was supple with immobility, and there was low muscle tension of the four limbs with positive and symmetric deep-tendon reflexes, which is a negative bilateral pathological sign. Lung auscultation revealed shortness of breath and extensive rales. Cardiac examination revealed normal heart sounds without murmurs and appropriate peripheral perfusion. The abdomen was soft without organomegaly.

Laboratory tests showed no obvious abnormalities in routine blood, urine, and stool tests. Autoantibodies for autoimmune encephalopathy and demyelinating diseases of the central nervous system, such as myelin oligodendrocyte glycoprotein, aquaporin-4, and myelin basic protein, were all negative. Syphilis, AIDS, and hepatitis were all negative. Lumbar puncture showed that cerebrospinal fluid pressure was normal with white blood cells $2 \times 10^{6} / \mathrm{L}$, protein $0.65 \mathrm{~g} / \mathrm{L}$, and lactate $2.6 \mathrm{mmol} / \mathrm{L}$; all cerebrospinal fluid cultures were negative.

On admission, chest $\mathrm{CT}$ showed bilateral pulmonary infection, especially in the inferior lobe (Fig. 1A). The brain magnetic resonance image (MRI) of the patient after admission is shown in Fig. 2C. Moreover, compared with MRIs conducted in 2012 (Fig. 2A) and 2016 (Fig. 2B), the patient had a trend in which the cerebellar sulci significantly widened along with severe cerebellar atrophy, thinning of the corpus callosum, and mild enlargement of lateral ventricle. Moreover, an electromyogram showed that normal nerve conduction velocity, and skin sympathetic reaction suggested autonomic neuropathy. An electroencephalogram after admission showed that during the awake period, a large number of bilateral symmetric 3-4c/s low and medium amplitude irregular slow waves were found, mainly in the anterior head and without obvious epileptiform waves (Fig. 3).

Whole-exome sequencing (WES) was employed to obtain a potential genetic diagnosis after admission. WES revealed compound heterozygous variants in the TECPR2 gene. Both variants were missense mutations. The first mutation was c. $1729 \mathrm{C}>\mathrm{T}$, causing an amino acid change

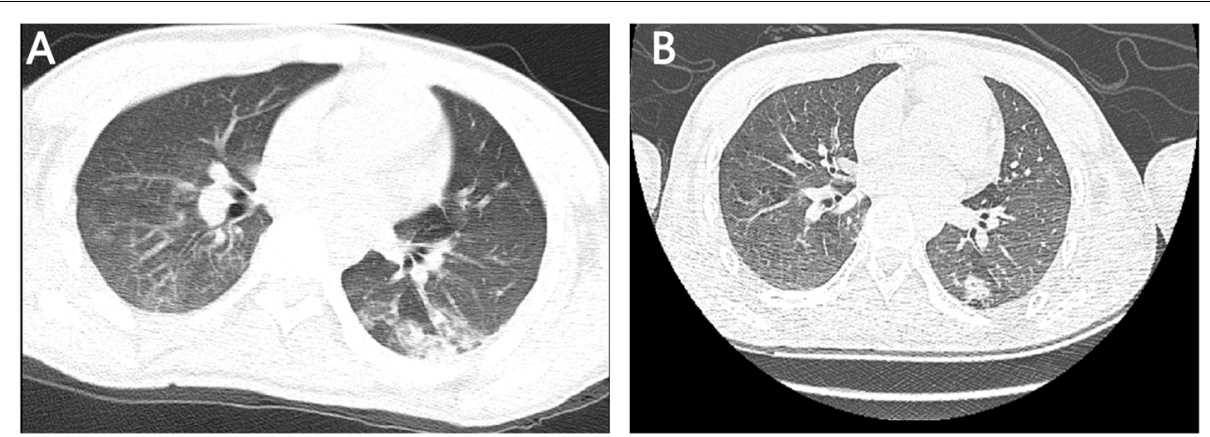

Fig. 1 Chest CT findings. After admission, the patient's chest CT showed bilateral pulmonary infection, especially in the inferior lobe (A). Eight days after admission, chest CT reexamination showed that bilateral pulmonary infection was controlled (B) 
A

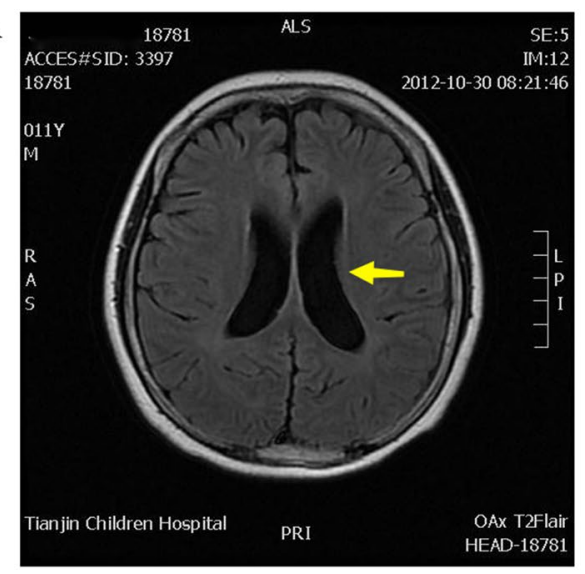

B

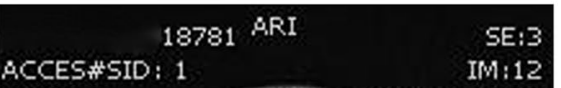

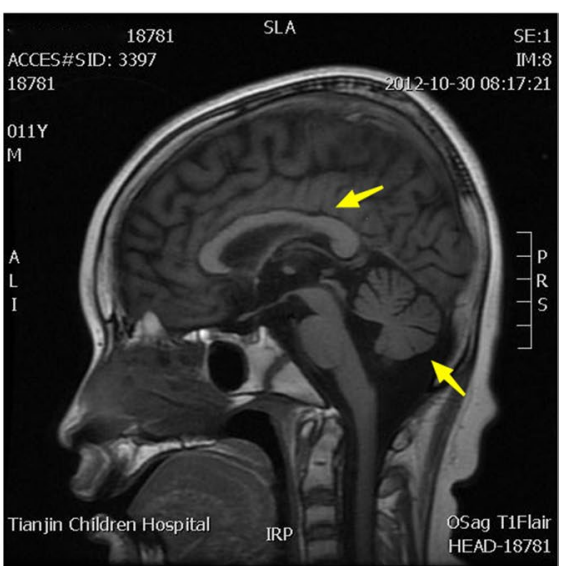
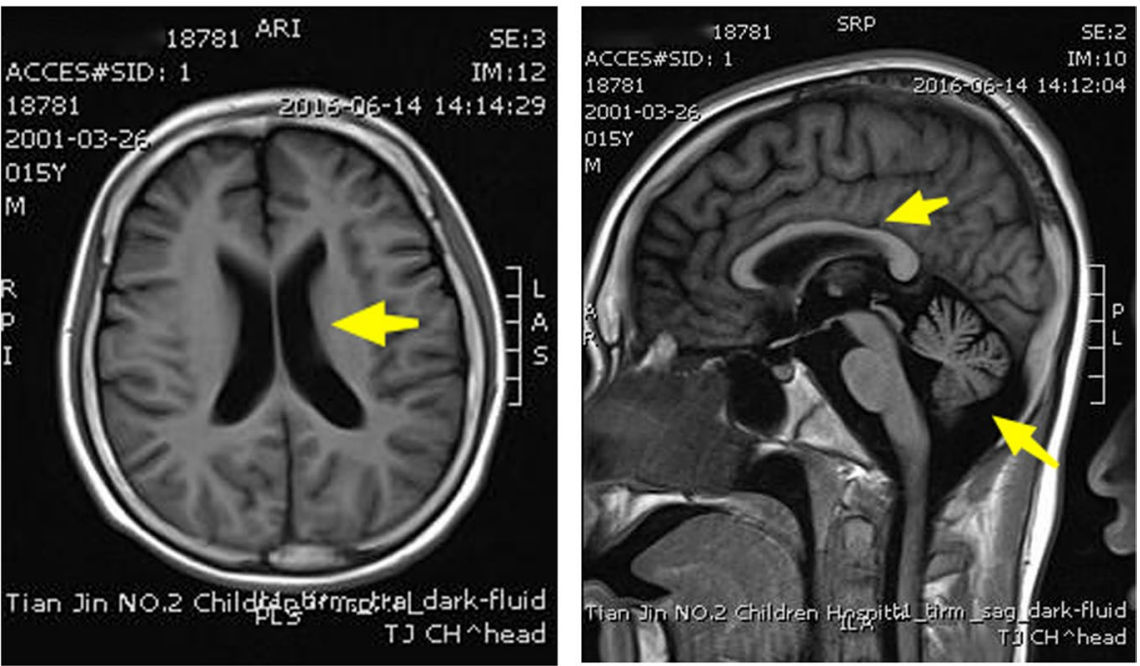

C
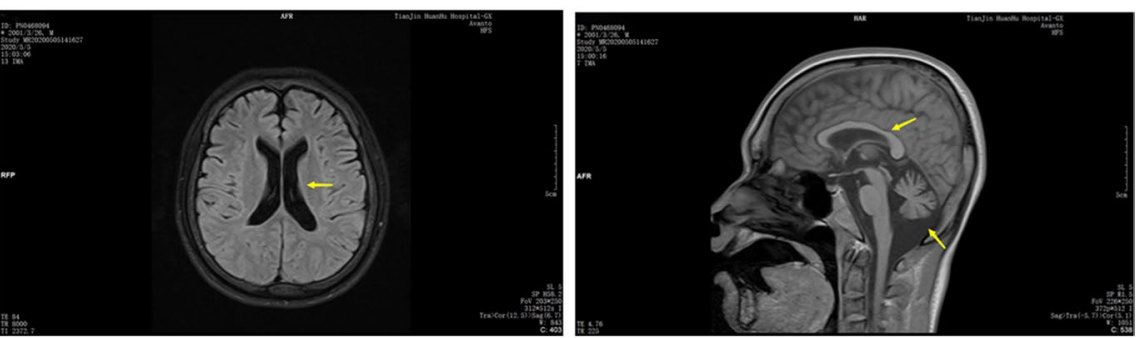

Fig. 2 Brain MRI findings. the brain MRI showed mild enlargement of lateral ventricle, corpus callosum was normal, and partial widening of the cerebellar sulci in 2012 (A); enlargement of lateral ventricle, partial thinning of corpus callosum, significant enlargement of the cerebellar sulci in 2016 (B); Cerebellar atrophy and thinning of corpus callosum were more serious than before in 2020 (C)

of p.H577Y (NM_014844.3) in the TECPR2 protein. The second missense variant was c.4189G $>\mathrm{A}$, causing the amino acid change p.A1397T (NM_014844.3). Neither mutation has been previously reported in patients with HSP49, and they are not currently in the HGMDpro database [8]. The mutation c.1729C $>$ T (p.H577Y) was inherited from the father, and c.4189G > A (p.A1397T) was inherited from the mother, which ultimately made the patient a compound heterozygote for these variants (Fig. 4).

After admission, the patient's oxygen saturation decreased intermittently, reaching a nadir of $70 \%$. The patient was treated with sputum suction and other airway nursing and cephalosporin antibiotics. After treatment, the infection was gradually controlled, consciousness turned clear, and blood oxygen saturation returned to normal. Eight days after admission, chest CT 


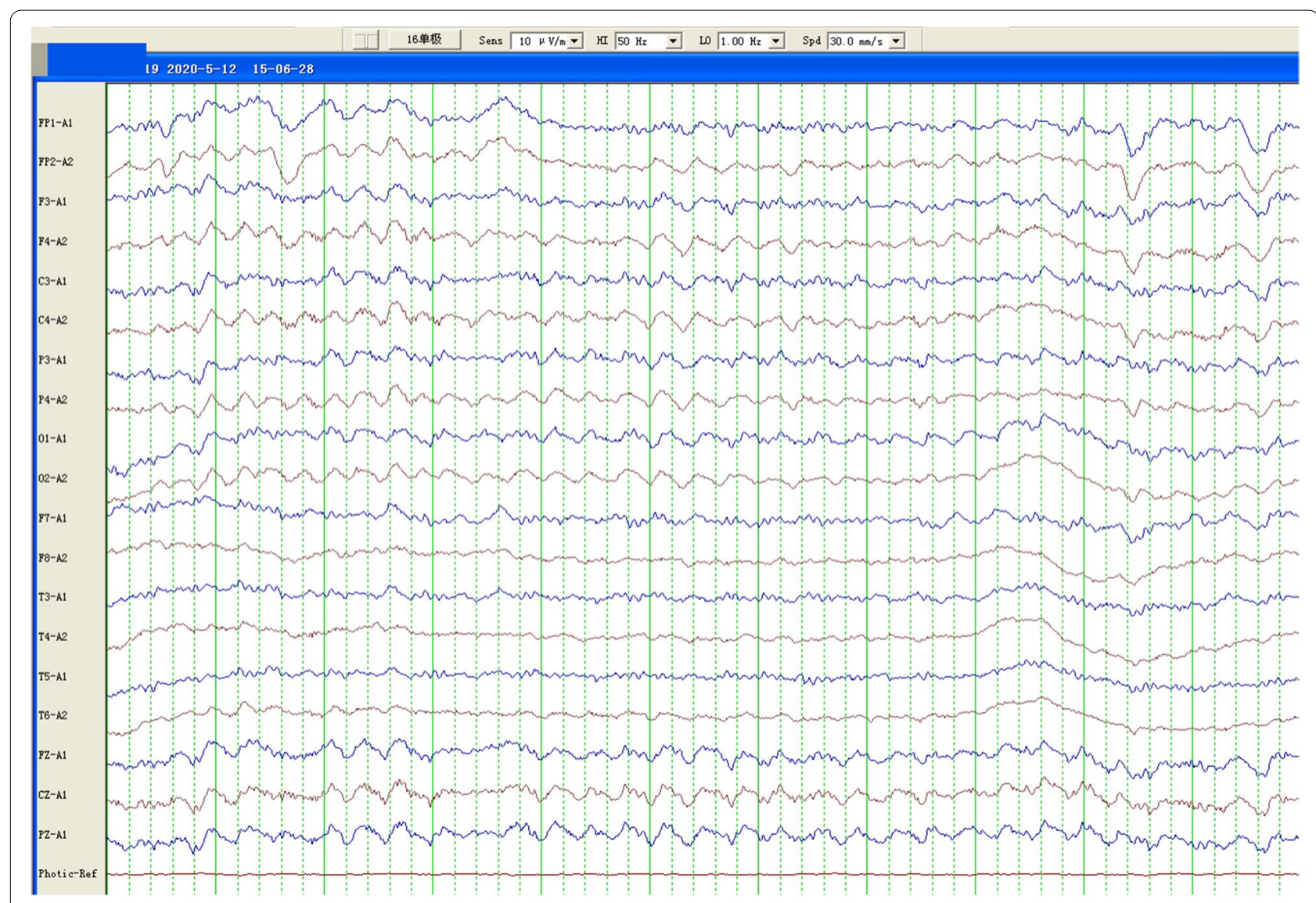

Fig. 3 Brain EEG findings. During the awake period, a large number of bilateral symmetric 3-4c/s low and medium amplitude irregular slow waves were found, mainly in the anterior head and without obvious epileptiform waves

reexamination showed that bilateral pulmonary infection was controlled and had improved (Fig. 1B).

During hospitalization, the patient's temperature increased paroxysmally, peaking at $38^{\circ} \mathrm{C}$. Routine blood work and procalcitonin levels were normal and the blood culture was negative, yet the patient's temperature still increased intermittently. At this time, the patient had no symptoms of infection and showed similar temperature fluctuations as those over the previous years before admission, which indicated possible autonomic dysregulation. The patient's digestive function was good after mental recovery, and gastroesophageal reflux disease was excluded after gastroenterology consultation. Sleep apnea syndrome was also excluded after sleep monitoring.

Moreover, seizures occurred recurrently after admission. Lamotrigine was provided to control seizures, and the onset frequency decreased until there was no epilepsy occurrence for up to 17 days. The patient was also provided idebenone to protect mitochondria, tizanidine to reduce muscle tension, and other nutritional nerve treatments.
The patient was discharged at 30 days of hospitalization. Discharge diagnosis was HSP49, secondary epilepsy, and pulmonary infection. At the time of discharge, the patient was conscious, aphasic, still had severe cognitive impairment and ataxic gait, and required walking assistance. Both upper limb tendon reflexes were ++ , left lower limb tendon reflex was + , right lower limb tendon reflexes were +++ , and bilateral pathological signs were not elicited. Follow-up was conducted 3 months later. The patient was prescribed lamotrigine and levetiracetam to control epilepsy, but he still had intermittent seizures, approximately once weekly.

\section{Discussion and conclusion}

HSP49 was first reported by Oz-Levi et al. in 2012 [5] as an autosomal recessive genetic disease mainly caused by TECPR 2 mutations. TECPR 2 is a binding partner of the mammalian Atg8 protein family and a possible positive regulator of autophagy [9-11]. Fraiberg et al. [12] showed that the protein encoded by TECPR 2 is involved in the targeting of autophagosomes to lysosomes; this process is impaired by mutations in the 
(A) TECPR2 c.1729C $>$ T (p.H577Y)

Patient

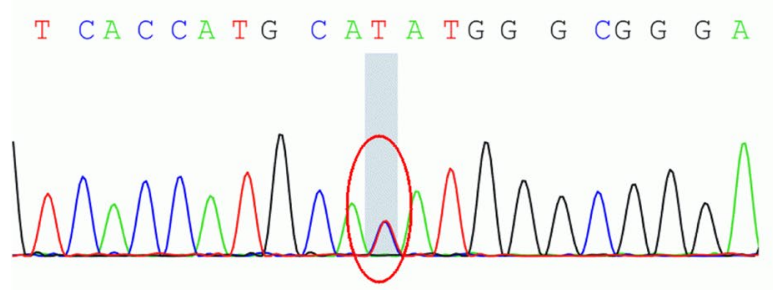

Father

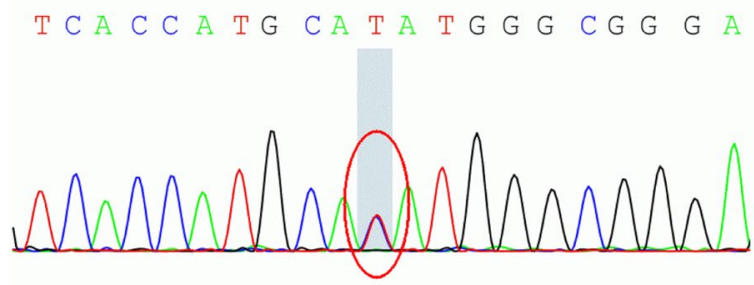

Mother

T C A C C A TG C A C A T G G G C G G G A

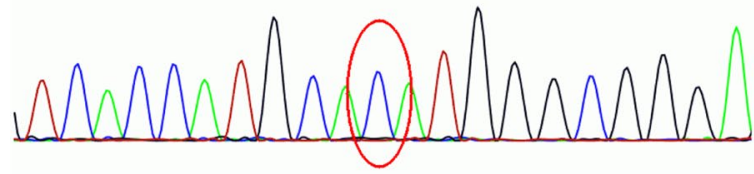

(B) TECPR2 c.4189G $>$ A (p.A1397T)

Patient

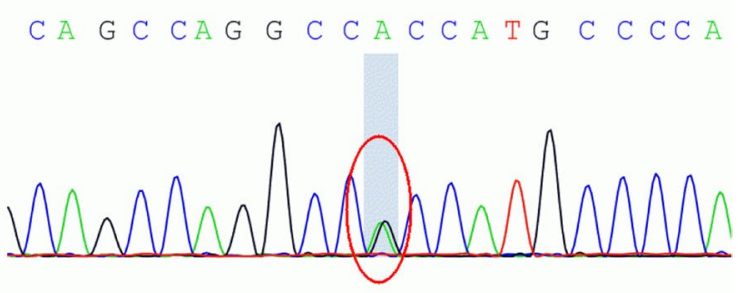

Father

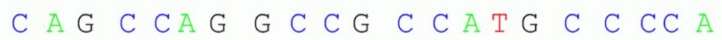

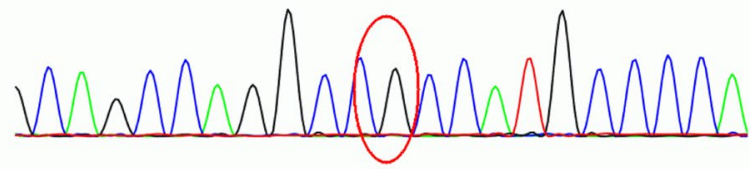

Mother

$C A G C C A G \quad G \quad C \quad C A C C A T G \quad C \quad C C C A$

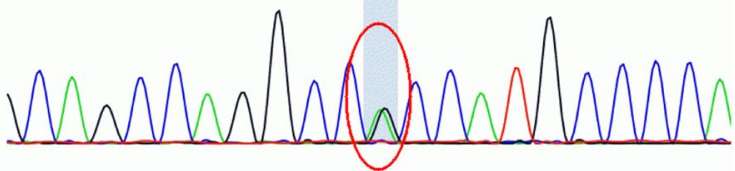

Fig. 4 Missense variants in the patient, his mother, and his father by WES. A variant c.1729C > T caused the amino acid change p.H577Y in TECPR2 and was inherited from the father. B variant c.4189G > A caused the amino acid change p.A1397T in TECPR2 and was inherited from the mother

TECPR2 gene, resulting in autophagosomes that cannot be eliminated and for which the accumulation leads to neurodegenerative diseases. To improve understanding of TECPR2 gene function, Tamim-Yecheskel et al. established a TECPR2 knockout mouse model. TECPR2 -/ - mice displayed clinical manifestations similar to those of HSP49 patients, including age-dependent neurodegenerative diseases. Moreover, abnormal accumulation of autophagosomes occurs in TECPR2 -/- mice in an age-dependent manner [13]. HSP49 displays clinical heterogeneity, which may be related to the diversity of TECPR2 gene mutation. Patients with HSP49 that harbor homozygous mutations exhibit obvious deformities, developmental delays, and hypotonia; these features typically manifest as mental retardation, spastic paraplegia, dysarthria, and ataxia. Characteristic dysmorphic features include microcephaly, dental crowding, a short broad neck, and a chubby appearance [5, $14]$.
Another clinical report regarding three children with mutations in TECRP2 also described outstanding features of sensory autonomic neuropathy that manifested as early hypotonia; areflexia; decreased pain and temperature sensitivity; and unstable blood pressure, body temperature, and osmotic pressure. The author proposed that the disease should therefore be classified as a new subtype of hereditary sensory-autonomic neuropathy, because in addition to intellectual disability and evolving spasticity, the main disabling feature of this unique disorder is autonomic-sensory neuropathy [6]. In contrast, clinical manifestations with heterozygous mutations are relatively mild, such as an Italian girl with HSP49 who displayed no impairment in mental development [7].

The patient in the present case also harbored heterozygous mutations and exhibited milder clinical symptoms than those in patients with homozygous mutations. The present patient had normal mental development and good athletic ability before the age of 
10 years. Although the patient showed central hypoventilation during lung infection, there was no dyspnea or hypoventilation after the lung infection was resolved. At present, the patient only presented with mental retardation, spastic ataxia, and paroxysmal convulsions. Moreover, the patient has paroxysmally high body temperature even in the absence of infection, indicating possible signs of autonomic dysregulation. The MRI of this patient is consistent with previous reports: severe cerebellar atrophy, enlargement of the fourth ventricle, thinning of the corpus callosum, and mild enlargement of lateral ventricle. Finally, the diagnosis of this patient was based on the detection of whole exons and two heterozygous variants in the exon region of the TECPR2 gene: c. $1729 \mathrm{C}>\mathrm{T}$ and c.4189G $>$ A. However, according to the HGMD pro database search, these two variant sites have not been previously reported. Although the pathogenicity of these two new mutations has not been determined, previous literature on the pathogenicity of other mutations in the TECPR 2 gene suggests $[5,7$, $14]$ that these two mutations are likely to be responsible for the TECPR2 gene abnormality and the present phenotype of the patients. At present, we only report the clinical manifestation and heterozygous variants in the TECPR2 gene of this patient. Future studies will further explore the pathogenicity of mutation of c.1729C $>\mathrm{T}$ and c.4189G $>\mathrm{A}$ in animal experiments.

The mild clinical manifestations of the present patient may be because the mutation site of the TECPR2 gene differs from those previously reported. However, as illustrated in animal models, the accumulation of autophagosomes caused by the abnormality of the TECPR2 is age-dependent; therefore, we will diligently follow up the patient's subsequent development, with particular attention to changes in patient's intelligence and muscle strength so that we can enact timely treatment.

The current case expands the gene mutation spectrum and clinical phenotypic characteristics of autosomal recessive HSP in China. In addition, this case shows that there are certain differences in the clinical manifestations of HSP49 in different ethnic groups. Moreover, it provides further evidence regarding the effectiveness of targeted next-generation sequencing technology in improving the efficiency and diagnostic rate of genetic diagnoses of HSP.

\footnotetext{
Abbreviations

HSP49: Hereditary spastic paraplegia 49; HSP: Hereditary spastic paraplegia; MRI: Magnetic resonance image; WES: Whole-exome sequencing.
}

\section{Acknowledgements}

We would like to thank the patient for his participation in this study.

\section{Authors' contributions}

YG: data collection, analysis and interpretation, drafting and revising the manuscript. WZ, XW, FL, KJ, RG, and HW: data collection. HL, SW, XW, ZS, and

YJ: analysis and interpretation. $Y G$ and SW: revising the manuscript. All authors have read and approved the final manuscript.

Funding

None.

\section{Availability of data and materials}

The datasets used and/or analysed during the current study are available from the corresponding author on reasonable request.

\section{Declarations}

Ethics approval and consent to participate

Not applicable

\section{Consent for publication}

The patient's guardian signed the written consent to publish this case report and any accompanying images. The copy of the consent form is available for review by the Editor of this journal.

\section{Competing interests}

Y. Guan reports no disclosures. H. Lu reports no disclosures. W. Zuo reports no disclosures. X. Wang reports no disclosures. S. Wang reports no disclosures. X. Wang reports no disclosures. F. Liu reports no disclosures. K. Jia reports no disclosures. R. Gao reports no disclosures. H. Wu reports no disclosures. Z. Shi reports no disclosures. Y. Ji reports no disclosures.

\section{Author details}

${ }^{1}$ Department of Neurology, Tianjin Huanhu Hospital, Tianjin 300350, China. ${ }^{2}$ Tianjin Key Laboratory of Cerebral Vascular and Neurodegenerative Disease, Tianjin 300350, China.

Received: 21 May 2021 Accepted: 25 January 2022

Published online: 07 February 2022

\section{References}

1. Adam MP, Ardinger HH, Pagon RA, et al., editors. GeneReviews [Internet]. Seattle (WA): University of Washington, Seattle; 1993-2022. Available from: https://www.ncbi.nlm.nih.gov/books/NBK1509/

2. Shimabukuro M, Hasegawa Y, Higa M, et al. Subclinical carotid atherosclerosis burden in the Japanese: comparison between Okinawa and Nagano residents. J Atheroscler Thromb. 2015;22:854-68.

3. Novarino G, Fenstermaker AG, Zaki MS, et al. Exome sequencing links corticospinal motor neuron disease to common neurodegenerative disorders. Science. 2014;343:506-11.

4. Husain RA, Grimmel M, Wagner M, et al. Bi-allelic HPDL variants cause a neurodegenerative disease ranging from neonatal encephalopathy to adolescent-onset spastic paraplegia. Am J Hum Genet. 2020;107(2):364-73.

5. Oz-Levi D, Ben-Zeev B, Ruzzo EK, et al. Mutation in TECPR2 reveals a role for autophagy in hereditary spastic paraparesis. Am J Hum Genet. 2012;91:1065-72.

6. Heimer G, Oz-Levi D, Eyal E, et al. TECPR2 mutations cause a new subtype of familial dysautonomia like hereditary sensory autonomic neuropathy with intellectual disability. Eur J Paediatr Neurol. 2016;20:69-79.

7. Covone AE, Fiorillo C, Acquaviva M, et al. WES in a family trio suggests involvement of TECPR2 in a complex form of progressive motor neuron disease. Clin Genet. 2016;90:182-5.

8. Stenson PD, Mort M, Ball EV, et al. The human gene mutation database: building a comprehensive mutation repository for clinical and molecular genetics, diagnostic testing and personalized genomic medicine. Hum Genet. 2014;133:1-9.

9. Behrends C, Sowa ME, Gygi SP, Harper JW. Network organization of the human autophagy system. Nature. 2010;466(7302):68-76. 
10. Stadel $D$, Millarte V, Tillmann KD, et al. TECPR2 cooperates with $L C 3 C$ to regulate COPII-dependent ER export. Mol Cell. 2015;60(1):89-104.

11. Ebrahimi-Fakhari D, Saffari $A$, Wahlster $L$, et al. Congenital disorders of autophagy: an emerging novel class of inborn errors of neuro-metabolism. Brain. 2016;139(Pt 2):317-37.

12. Fraiberg M, Tamim-Yecheskel BC, Kokabi K, et al. Lysosomal targeting of autophagosomes by the TECPR domain of TECPR2. Autophagy. 2021;17(10):3096-108.

13. Tamim-Yecheskel BC, Fraiberg M, Kokabi K, et al. A tecpr2 knockout mouse exhibits age-dependent neuroaxonal dystrophy associated with autophagosome accumulation. Autophagy. 2021;17(10):3082-95.

14. Zhu X, Petrovski S, Xie P, et al. Whole-exome sequencing in undiagnosed genetic diseases: interpreting 119 trios. Genet Med. 2015;17:774-81.

\section{Publisher's Note}

Springer Nature remains neutral with regard to jurisdictional claims in published maps and institutional affiliations.

- fast, convenient online submission

- thorough peer review by experienced researchers in your field

- rapid publication on acceptance

- support for research data, including large and complex data types

- gold Open Access which fosters wider collaboration and increased citations

- maximum visibility for your research: over 100M website views per year

At BMC, research is always in progress.

Learn more biomedcentral.com/submissions 